Bio - grafía. Escritos sobre la Biología y su Enseñanza. ISSN 2027-1034

Edición Extraordinaria. p.p. 1034 - 1042

Memorias del IX Encuentro Nacional de Experiencias en Enseñanza de la Biología y la Educación Ambiental. IV Congreso Nacional de Investigación en Enseñanza de la Biología.

\title{
TRABAJAR EL PASSAJE ATRAVÉS DE LOS CINCO SENTIDOS: EVALUACIÓN DE UN MATERIAL PARA LA INNOVACIÓN EDUCATIVA
}

WORKING THE LANDSCAPE THROUGH THE FIVE SENSES: EVALUATION OF A MATERIAL FOR EDUCATIONAL INNOVATION

\author{
Olga Mayoral García-Berlanga \\ Marta Talavera \\ Amparo Hurtado ${ }^{1}$
}

\section{RESUMEN}

Se presenta la evaluación de un material didáctico que permite abordar la percepción del paisaje a través de los cinco sentidos. Este material está constituido por una batería de propuestas didácticas que pueden trabajarse de manera aislada 0 secuenciada, realizando todas las actividades propuestas. Las propuestas incluyen actividades que abordan la percepción de diferentes tipos de paisaje a través un solo sentido y otras que implican el estímulo de varios sentidos simultáneamente (actividades mixtas). El primer material elaborado fue sometido al juicio de 6 expertos/as en el diseño de materiales educativos y familiarizados con la temática del paisaje, lo que permitió introducir mejoras en el diseño y contenidos. La segunda versión del cuaderno didáctico fue aplicada en un taller piloto con alumnado de Educación Primaria, que ayudó a evidenciar sus preferencias y dificultades en el uso de los materiales propuesto, así como a la evaluación por parte de futuros docentes de Infantil y Primaria: estudiantes de $2^{\circ}$ y $4^{\circ}$ curso del Grado en Maestro/a en Educación Primaria/Infantil, de la Facultad de Magisterio de la Universitat de València (España).

PALABRAS CLAVE: paisaje, material didáctico, competencias básicas, Infantil, Primaria, sentidos

\section{ABSTRACT}

The paper presents the evaluation of teaching materials that can address the perception of the landscape through the five senses. This material consists of a set of educational proposals that can be worked isolated or sequenced, making all the proposed activities. Proposals include activities that address the perception of different types of landscape through a single sense and others that involve the stimulation of several senses simultaneously (mixed activities). The first version of the material was subjected to the judgment of 6 people with expertise in the design of educational and familiar with the topic of landscapes, enabling improvements in design and content. The second version of the teaching materials was applied in a pilot workshop with students of Primary School, which helped highlight the preferences and difficulties of students in the use of the proposed materials, as well as the evaluation by future

\footnotetext{
${ }^{1}$ Universitat de Valencia, España
} 
Bio - grafía. Escritos sobre la Biología y su Enseñanza. ISSN 2027-1034

Edición Extraordinaria. p.p. 1034 - 1042

Memorias del IX Encuentro Nacional de Experiencias en Enseñanza de la Biología y la Educación Ambiental. IV Congreso Nacional de Investigación en Enseñanza de la Biología.

teachers: students of $2^{\text {nd }}$ and $4^{\text {th }}$ year of the Degree in Preschool and Primary School Education of the Faculty of Education of the Universitat de València (Spain).

KEY WORDS: landscape, teaching materials, basic competences Preschool, Primary School, senses

\section{INTRODUCCIÓN}

La educación del futuro debe capacitar al alumnado para conectar con su realidad inmediata, compleja y cambiante, con aquella en que los ciudadanos deberán desarrollar sus proyectos vitales futuros. El uso del paisaje permite la adquisición de competencias básicas a través de la percepción directa e indirecta del territorio (Hernández, 2010). Esto responde a lo que se recoge en el proyecto de la OCDE, Definición y Selección de Competencias (DeSeCo, 2002); "una competencia es más que conocimiento y destrezas", supone desarrollar la capacidad de responder a demandas complejas y llevar a cabo tareas diversas de forma adecuada. En concreto la competencia de Conocimiento e interacción con el mundo físico, estaría asociada con la percepción y conocimiento del espacio físico, con la comprensión de la interrelación existente entre el medio natural y las actividades humanas, que da lugar a esa gran pluralidad de paisajes. Esta competencia debe incentivar el uso responsable de los recursos naturales y el cuidado del medio ambiente.

Esto puede utilizarse como objetivo de etapa, tanto a nivel de la Educación Infantil como Primaria, contribuyendo no sólo a determinar las características y elementos de los paisajes, sino también a trabajar, tanto de manera individual como en grupo, procedimientos y destrezas, así como a fomentar valores. Para realizar esta adaptación de una manera sistemática, es necesario el desarrollo de materiales de innovación educativa que capaciten al profesorado y presenten una estructura didáctica con un nuevo enfoque de enseñanza, de forma que sea un material de apoyo para el área, que permita realizar innovaciones destinadas a enseñar al educando, a utilizar los conocimientos y a su vez a desarrollar su creatividad.

Para comprobar si los materiales diseñados se ajustan a los principios y al diseño curricular planteados y a la metodología de la reforma educativa, adecuándose al contexto y al alumnado, y por supuesto a los planteamientos de los propios centros, es necesaria una evaluación de los mismos (Sevillano, 1995). Esta evaluación adquiere más sentido, si cabe, cuando es de elaboración propia, por la necesidad de saber si lo que se ha diseñado es válido y útil en relación a su finalidad. Una evaluación inicial permite realizar los cambios necesarios antes de su aplicación y un análisis positivo debería asegurar la contribución a la innovación educativa en la búsqueda de la mejora del proceso de enseñanza-aprendizaje. Para asegurar la efectividad de esta evaluación, deben participar todos los actores implicados en su elaboración y uso; es decir, los diseñadores, el profesorado que va a utilizarlo y el alumnado al que va dirigido. De esta forma se obtendrán diferentes visiones sobre unos mismos materiales que, complementándose entre sí, podrán proporcionar una evaluación lo más objetiva posible.

Tampoco se puede perder de vista el objeto sobre el que se pretende realizar el aprendizaje, en este caso, el paisaje, ni el modo en el que pretende que éste se 


\section{Bio - grafía. Escritos sobre la Biología y su Enseñanza. ISSN 2027-1034}

Edición Extraordinaria. p.p. 1034 - 1042

Memorias del IX Encuentro Nacional de Experiencias en Enseñanza de la Biología y la Educación Ambiental. IV Congreso Nacional de Investigación en Enseñanza de la Biología.

alcance, en este caso, a través de un aprendizaje emocional de forma que la noción del paisaje se construya de manera significativa, a partir de diversos tipos de información sensorial, afectiva y perceptiva.

Para este aprendizaje se define el paisaje como "cualquier parte del territorio, tal y como es percibida por las poblaciones, cuyo carácter resulta de la acción de factores naturales y/o humanos y de sus interrelaciones" según apunta el Convenio Europeo de Paisaje (Consejo de Europa. Florencia, 2000). Con esta definición, el aprendizaje de este concepto supone una combinación de habilidades prácticas, conocimientos, motivación, valores éticos, actitudes, emociones y otros componentes sociales y de comportamiento que se movilizan conjuntamente para lograr una acción eficaz e innovadora.

La intención de un aprendizaje emocional, implicaría, partiendo de este concepto de paisaje, más allá de lo físico, reflexionar acerca de su vertiente más emocional, definiéndolo como una entidad cultural subjetiva, simbólica y cambiante (Howes, 2003). Esto se puede conseguir con paisajes próximos al alumnado, adaptados a su contexto sociocultural, y que despierten recuerdos y sensaciones de proximidad, y con el acercamiento a dichos paisajes a través de los cinco sentidos, ya que este análisis perceptivo del paisaje, partiendo de la subjetividad del mismo, permite descubrir determinados comportamientos ante éste, y desarrollar una actitud crítica y tolerante, intentando abordar la complejidad del fenómeno estudiado (Souto, 1998; Martínez, 2013). Es decir, que el hecho de haberlo visto, tocado, olido, pisado, oído, degustado, conducirá a una mejor afinidad, una mayor proximidad.

Así pues, se presenta la evaluación de un material curricular, para ser utilizado en el aula de Infantil y Primaria (Talavera et al., 2015), con el que el profesorado puede afrontar la enseñanza-aprendizaje del paisaje próximo, sus características e implicaciones, a través de un acercamiento sensorial al mismo.

\section{METODOLOGÍA}

El material que se presenta se elaboró en una fase previa de diseño, basada en la recopilación de documentación que respondiera a la necesidad docente de materiales que abordara la competencia de interacción con el mundo físico, y en concreto con el paisaje próximo (DOCV, 2014). Para responder a esta necesidad, se elaboró un material didáctico, organizado en sesiones prácticas, y que contuviera los elementos necesarios mínimos para el desarrollo de esta competencia, mediante aportaciones y opiniones de expertos en la materia y que así mismo fuera útil para los docentes de Educación Primaria e Infantil y atractivo para los usuarios finales, que son los estudiantes. Para ello se procedió a investigar acerca de la realización de material didáctico, su diseño y valoración, consultando en distintas fuentes.

En base a la información recabada y a la experiencia previa de las autoras en el desarrollo de propuestas educativas en cuestiones ambientales, se diseñó una guía didáctica, compuesta por un conjunto de actividades, estructuradas en fichas independientes fotocopiables, centradas en el desarrollo de los cinco sentidos, tanto de manera independiente como en actividades mixtas que combinan dos o más sentidos, siempre en relación con el paisaje. La propuesta incluía un juego de cartas para la identificación de algunas especies de flora autóctona. Un apartado final recogía 
Bio - grafía. Escritos sobre la Biología y su Enseñanza. ISSN 2027-1034

Edición Extraordinaria. p.p. 1034 - 1042

Memorias del IX Encuentro Nacional de Experiencias en Enseñanza de la Biología y la Educación Ambiental. IV Congreso Nacional de Investigación en Enseñanza de la Biología.

un listado de los materiales para cada actividad. La duración aproximada de las actividades es de unos 20 minutos cada una y pueden aplicarse con alumnado de entre 6 y 12 años (y en la mayor parte de los casos también con alumnado de menos de 6 años).

El primer material elaborado se sometió al juicio de 6 expertos/as en la temática del paisaje y en el diseño de este tipo de materiales, que valoraron una primera versión del material didáctico, aportando modificaciones tanto a nivel de diseño como de contenidos.

Una vez incorporadas dichas modificaciones, se elaboró una segunda versión del material didáctico y se diseñó una sesión piloto con formato de taller, de una hora de duración, con alumnado de Primaria (28 alumnos/as de un centro público de la ciudad de Valencia). Durante dicha sesión, el equipo de investigación tomó nota de las preferencias y dificultades del alumnado, a nivel de uso de los recursos materiales propuestos para el desarrollo de las actividades según la guía didáctica.

Por último, se sometió el material a una tercera evaluación por parte futuros docentes en formación, estudiantes de $2^{\circ}$ y $4^{\circ}$ curso del Grado en Maestro/a en Educación Primaria/Infantil, de la Facultad de Magisterio de la Universitat de València.

Para esta evaluación se diseñó una rúbrica en la que se valoraban los aspectos visuales, de contenidos, materiales, interés de las actividades (para el alumnado), y aspectos del desarrollo de cada una de las actividades reflejadas en la guía, puntuando las siguientes afirmaciones mediante una escala Likert de 0-10 (donde 0 es "totalmente en desacuerdo" y 10 "totalmente de acuerdo"). También se añadieron tres preguntas abiertas para valorar el conjunto de las actividades, recoger propuestas de mejora y resaltar los aspectos más interesantes, así como una última valoración global de dichas actividades en formato numérico (del 1 al 10).y una indicación de a qué curso podrían ir dirigidas estas actividades (anexo I).

Durante una sesión de trabajo con grupos de unas 25 personas, se presentó el material elaborado, junto a los materiales físicos que se proponían para el desarrollo del mismo en las aulas y se solicitó a cada participante la valoración de cinco de las actividades que se proponían. Esta sesión se repitió en tres ocasiones (con 25, 21 y 24 participantes cada vez).

\section{RESULTADOS Y DISCUSIÓN}

\section{Resultados de la evaluación}

Una vez aplicadas todas las modificaciones, se obtuvo el cuaderno titulado "Descubriendo el paisaje con los cinco sentidos. Material didáctico fotocopiable", fue editado (Talavera et al., 2015), y se viene aplicando con alumnado de diferentes niveles educativos. Este cuaderno constituye una guía didáctica compuesta por 25 actividades, agrupadas en función de los sentidos implicados en ellas. Así mismo incluye un juego de cartas destinado a aproximar al alumnado al entorno vegetal más inmediato, de un modo lúdico y participativo. Previamente a esta utilización se sometió al proceso de evaluación que se describe en la metodología. 
Bio - grafía. Escritos sobre la Biología y su Enseñanza. ISSN 2027-1034

Edición Extraordinaria. p.p. 1034 - 1042

Memorias del IX Encuentro Nacional de Experiencias en Enseñanza de la Biología y la Educación Ambiental. IV Congreso Nacional de Investigación en Enseñanza de la Biología.

A continuación se detallan los resultados de la rúbrica de evaluación realizada a profesores en formación de la Facultad de Magisterio de la Universidad de Valencia., para ello se utilizó una muestra de 162 futuros docentes, 27 de $4^{\circ}$ curso y 135 de segundo curso, 91 mujeres y 71 hombres.

El $54,3 \%$ de los encuestados destacan que las actividades deberían encuadrarse en un nivel de 3-4을 de primaria, mientras que nadie cree que serían apropiadas para el nivel infantil y sólo un 2,5\% las utilizaría en niveles superiores (tabla 1).

Tabla 1 Respuestas a la pregunta ¿En qué curso de primaria encuadrarías esta actividad?

\begin{tabular}{|c|c|c|}
\hline Nivel & $\mathbf{N}$ & $\%$ \\
\hline Infantil & 0 & 0 \\
\hline $1^{\circ}$ Primaria & 15 & 9,3 \\
\hline $2^{\circ}$ Primaria & 12 & 7,4 \\
\hline 1er ciclo & 7 & 4,3 \\
\hline 3ํ Primaria & 48 & 29,6 \\
\hline $4^{\circ}$ Primaria & 40 & 24,7 \\
\hline $2^{\circ}$ Ciclo & 12 & 7,4 \\
\hline 5 Primaria & 14 & 8,6 \\
\hline 6을 Primaria & 2 & 1,2 \\
\hline 3er ciclo & 4 & 2,5 \\
\hline \multirow[t]{2}{*}{$\mathrm{Ns} / \mathrm{Nc}$} & 8 & 5 \\
\hline & 162 & 100 \\
\hline
\end{tabular}

Respecto a la valoración de cada una de las actividades, todas ellas obtienen una media superior a 5 puntos, destacando las actividades Auditiva 5 y Gusto 1 que obtienen la máxima puntuación.

Respecto a los aspectos visuales, la actividad Visual 2 y la Gusto-Olfato destacan por su calidad, frente a la Auditiva 2 que no gustó tanto y es valorada con un 5.65 (Tabla 2). 
Bio - grafía. Escritos sobre la Biología y su Enseñanza. ISSN 2027-1034

Edición Extraordinaria. p.p. 1034 - 1042

Memorias del IX Encuentro Nacional de Experiencias en Enseñanza de la Biología y la Educación Ambiental. IV Congreso Nacional de Investigación en Enseñanza de la Biología.

Tabla 2. Valoraciones globales

\begin{tabular}{|c|c|c|c|c|c|}
\hline \multicolumn{2}{|c|}{ Actividad N } & \multirow{3}{*}{$\begin{array}{l}\begin{array}{l}\text { Valoració } \\
\text { n global }\end{array} \\
6,66\end{array}$} & \multirow{3}{*}{$\begin{array}{l}\mathrm{O} 1 \\
\mathrm{O} 2\end{array}$} & \multirow{2}{*}{$\begin{array}{l}31 \\
35\end{array}$} & \multirow{3}{*}{$\begin{array}{l}7,58 \\
7,14 \\
6,53\end{array}$} \\
\hline & & & & & \\
\hline$\overline{\mathrm{A} 1}$ & 34 & & & 34 & \\
\hline A2 & 34 & 5,65 & O4 & 30 & 7,35 \\
\hline A3 & 30 & 7,18 & T1 & 32 & 7,21 \\
\hline A4 & 34 & 5,83 & T2 & 34 & 7,51 \\
\hline A5 & 31 & 8,14 & T3 & 32 & 6,9 \\
\hline G1 & 34 & 8,26 & V1 & 33 & 7,78 \\
\hline G2 & 34 & 7,47 & V2 & 34 & 7,46 \\
\hline G3 & 31 & 7,37 & V3 & 32 & 7,61 \\
\hline GO & 32 & 8 & V4 & 35 & 7,72 \\
\hline GT & 33 & 7,58 & V5 & 30 & 7,05 \\
\hline GV & 33 & 6,53 & Total & 781 & \\
\hline $\mathrm{JP}$ & 29 & 7,86 & & & \\
\hline
\end{tabular}

Entre las actividades en las que mejor se trabajan los contenidos propuestos, las actividades auditivas superan al resto al ser valoradas por los participantes, mientras que las actividades visuales tienen un mayor interés. En el desarrollo de las actividades, no se aprecian diferencias entre los diferentes grupos de actividades. El juego de cartas es valorado con una media de 7,8 puntos, frente a 10.

En las respuestas a las preguntas abiertas, la primera cuestión, en la que se preguntaba por la opinión general del conjunto de actividades y materiales de apoyo, los encuestados destacan su interés, originalidad y contenido lúdico, que, según la opinión de los encuestados, facilitaría el tratamiento de estos contenidos de una manera entretenida, amena y diferente, facilitando el desarrollo de la creatividad y motivación del alumnado.

Entre los aspectos que mejorarían, recogidos en la segunda pregunta, destacan la necesidad de introducir colores más animados en las ilustraciones que acompañan a las actividades, la posibilidad de reducir algunos textos y adaptarlos a niveles inferiores y destacan que deben ir acompañadas de una explicación por parte del docente para la total comprensión de las mismas.

Los aspectos más interesantes para los encuestados, según lo recogido en la tercera pregunta, eran la variedad de actividades, la introducción de actividades relacionadas con el olfato, la utilización de materiales próximos y fáciles de adquirir y la presentación de actividades mixtas, en las que se confluyen varios sentidos. 
Bio - grafía. Escritos sobre la Biología y su Enseñanza. ISSN 2027-1034

Edición Extraordinaria. p.p. 1034 - 1042

Memorias del IX Encuentro Nacional de Experiencias en Enseñanza de la Biología y la Educación Ambiental. IV Congreso Nacional de Investigación en Enseñanza de la Biología.

\section{CONCLUSIONES}

Se ha presentado la evaluación de un material con un diseño encaminado a ser utilizado en el aula de Primaria, con el que el profesorado puede afrontar la enseñanza-aprendizaje del paisaje próximo, sus características e implicaciones, a través de un acercamiento sensorial.

Este material ha sido sometido a una evaluación inicial por parte de expertos y ha sido aplicada en una prueba piloto con alumnado de Primaria con resultados satisfactorios. Actualmente se está desarrollando una evaluación pormenorizada en la que se implica al profesorado en formación. El objetivo final de esta evaluación ha de ser avalar su calidad y cumplir los objetivos propuestos de desarrollo de la competencia básica de interacción con el medio de una manera lúdica, motivadora e innovadora.

El cuaderno didáctico aquí presentado, ya editado y que está siendo aplicado en las aulas sigue en continuo progreso, de modo que todos aquellos aspectos que la práctica y aplicación prolongadas muestran que pueden ser mejorables, serán incorporados en futuras ediciones. Se trata, por lo tanto de una propuesta en continua evolución, que dota a la iniciativa de dinamismo.

Diversos autores han revisado las posibilidades didácticas del paisaje desde un entorno de aprendizaje constructivista, como medio educativo idóneo, incluyendo el paisaje como recurso didáctico que ofrece dos variaciones: escenario geográfico y secuencia geográfica (García de la Vega, 2012). Este enfoque geográfico coincide con la idea de Martínez de Pisón (2009) de que "el paisaje es la manifestación formal de la realidad geográfica". Sin embargo, el paisaje ha sido escasamente trabajado como recurso educativo desde las ciencias naturales o desde un punto de vista sensorial y del aprovechamiento de las vivencias y percepciones que provoca en el alumnado.

La propuesta del cuadernillo pretende enriquecer la percepción del paisaje, ampliándola en dos ámbitos: por una parte, explorando su percepción sensorial y por otra, acercando al alumnado a una vivencia más biológica, en relación con los organismos vivos que conforman y modelan el paisaje, incluyendo la acción del ser humano. En este último aspecto, viene a coincidir con el abordaje desde la geografía, que considera las interrelaciones humanas en el territorio y que debe potenciar actitudes positivas y compromisos, partiendo del conocimiento del paisaje.

Atendiendo al currículo, resulta llamativo que el paisaje sólo es abordado desde el Área de las Ciencias Sociales, considerando que "la geografía aporta una sensibilización hacia el valor de los paisajes y el uso y conservación de los recursos ambientales para lograr un desarrollo sostenible y justo". Se trabaja dentro del bloque 2 (El mundo en que vivimos), en todos los cursos, aunque también es abordado en el $5^{\circ}$ curso, en el Área de Cultura Valenciana, en el bloque 1 (El espacio geográfico valenciano). Si bien el estudio del paisaje desde este enfoque geográfico "contribuye a comprender las interacciones entre los fenómenos naturales y las actividades humanas que explican las transformaciones de los espacios y regiones", por lo que es considerado en el mismo currículo oficial como "puente" hacia las Ciencias Naturales, entendemos que propuestas como la aquí presentada, contribuyen de manera destacable a completar el concepto de paisaje. El paisaje ofrece la posibilidad de propiciar que los contenidos curriculares resulten atractivos y manejables, organizados en torno a ciertos temas, extraídos del mundo real, que resulten atractivos, tanto para docentes y alumnos (Beane, 2005; Bruner, 2008). 
Bio - grafía. Escritos sobre la Biología y su Enseñanza. ISSN 2027-1034

Edición Extraordinaria. p.p. 1034 - 1042

Memorias del IX Encuentro Nacional de Experiencias en Enseñanza de la Biología y la Educación Ambiental. IV Congreso Nacional de Investigación en Enseñanza de la Biología.

La propuesta didáctica aquí presentada, si bien fue diseñada para Primaria, es fácilmente transferible a estudiantes de segundo ciclo de Educación Infantil, mediante la adaptación de parte de los materiales. En este sentido, cabe destacarse que en el propio currículo de infantil se aborda el paisaje. Concretamente en los bloques 2 (El acercamiento a la naturaleza) y 3 (La cultura y vida en sociedad), en los que se incluye la identificación de distintos tipos de paisaje: rural y urbano, así como las diferentes formas de organización humana en ellos.

El interés reciente de las instituciones europeas en relación al paisaje y su conservación, debe tener su repercusión en las aulas, lo que ha de conseguirse mediante propuestas atractivas. En este sentido, el cuadernillo "Descubriendo el paisaje con los cinco sentidos" pretende contribuir a la revalorización del paisaje como elemento a conservar, así como recurso didáctico de primer orden.

\section{BIBLIOGRAFIÁ}

Alvarez-Suárez R. (2003) La utilización de modelos experimentales en geología. Alambique. Didáctica de las Ciencias Experimentales 35, 60-69.

Bolacha E., Deus H. M., Caranova R., Costa A. M., Silva S., Vicente J., Fonseca P. E. (2006) Uma experiência na formação de professores: Modelação Analógica de Fenómenos Geológicos - A Geologia no laboratório. Geonovas 20, 33-56.

Bueno E. (2004) Aprendiendo química en casa. Revista Eureka sobre Enseñanza y Divulgación de las Ciencias 1 (1), 45-51.

Castilho Razera J.C. (2015) Un perfil cienciométrico de Revista Eureka sobre Enseñanza y Divulgación de las Ciencias (2004-2013). Revista Eureka sobre Enseñanza y Divulgación de las Ciencias 12 (2), 237-248. http://hdl.handle.net/10498/17249

Davies I. (2004) Science and citizenship education. International Journal of Science Education 26 (14), 1751-1763.

Edge R. D. (1982) Physics and the new games, or pretend you're an atom, The Physics Teacher 20, 596-601.

Grilli J., Laxague M., Barboza L. (2015) Dibujo, fotografía y Biología. Construir ciencia con y a partir de la imagen. Revista Eureka sobre Enseñanza y Divulgación de las Ciencias 12 (1), 91-108. http://hdl.handle.net/10498/16926

Martínez de Sousa J. (2008) Secc. 10.5.8.2 en Ortografía y ortotipografía del español actual, 2. ${ }^{\text {a }}$ ed., corregida. Somonte-Cenero: Ediciones Trea.

Merino J., Herrero F. (2007) Resolución de problemas experimentales de Química: una alternativa a las prácticas tradicionales. Revista Electrónica de Enseñanza de las Ciencias 6 (3), 630-648.

Ribeiro A. (2008) Tectónica de placas, pp. 5-6 en Mateus A. (coord.), O interior da terra: da crusta ao núcleo. Lisboa. Departamento de Geologia FCUL. 
Bio - grafía. Escritos sobre la Biología y su Enseñanza. ISSN 2027-1034

Edición Extraordinaria. p.p. 1034 - 1042

Memorias del IX Encuentro Nacional de Experiencias en Enseñanza de la Biología y la Educación Ambiental. IV Congreso Nacional de Investigación en Enseñanza de la Biología.

Roth W., Désautels J. (2004) Educating for citizenship: Reappraising the role of science education. Canadian Journal of Science, Mathematics and Technology Education 2 (4), 149-168.

Talavera, M., Mayoral, O., Hurtado, A., Botella, A.M., Martínez, S. (2015) Descubriendo el paisaje con los cinco sentidos. Material Didáctico Fotocopiable. ISBN: 978-84-608-3644-5. 